\title{
Madame de Maintenon et la tradition humaniste dans l'éducation des demoiselles de Saint-Cyr
}

\author{
Strosetzki, Christoph
}

First published in:

Niderst, Alain (ed.): Autour de Françoise d'Aubigné, Marquise de Maintenon : actes des journées de Niort, 23 - 25 mai 1996, tome II. (Albineana 10/11,2). Paris : Champion, 1999, p. $425-446$

ISSN 1154-5852 


\section{MADAME DE MAINTENON \\ ET LA TRADITION HUMANISTE \\ DANS L'ÉDUCATION DES DEMOISELLES DE \\ SAINT-CYR}

Le principal souci des humanistes français fut d'encourager la lecture de livres profanes de l'Antiquité ainsi que les commentaires et traductions critiques de textes bibliques et pieux. On aspirait à l'essor encyclopédique des connaissances jusqu'alors acquises. La curiosité était la vertu fondamentale. Montaigne exigeait de ses lecteurs qu'ils aient le goût pour la curiosité, à savoir une « continuelle exercitation à remarquer les choses incogneues et nouvelles ", "afin qu'ayant en l'imagination cette continuelle variation des choses humaines, nous en ayons le jugement plus esclaircy et plus ferme ${ }^{2}$. Au XVII siècle, les jansénistes et les quiétistes venaient de faire leur apparition. La question est alors de savoir si l'éducation religieuse, qu'encourageait $\mathbf{M}^{\text {me }}$ de Maintenon, répondait à l'aube du siècle des Lumières au postulat sur la curiosité de Montaigne $e^{3}$, ou sollicitait au contraire, dans l'intérêt de garantir l'orthodoxie, une position analogue à celle de l'humaniste espagnol Vives, qui, compte tenu de l'influence luthérienne et calviniste au $\mathrm{XVI}^{\mathrm{e}}$ siècle, voulait refréner la curiosité et favoriser la censure ${ }^{4}$.

Si l'on prend en considération que les humanistes, comme les grammatici du Moyen Age dans le cadre du trivium des artes liberales, instruisent leurs élèves en latin en établissant un catalogue

1. M. de Montaigne, Euvres complètes, (éd.) M. Rat, Paris 1962, Gallimard, p. 951. 2. Ibid., p. 285.

3. Cf. Chr. Strosetzki, Verstehen bei Montaigne, dans Wolfenbütteler Renaissance Mitteilungen 6/2, 1982, p. 89-104.

4. Cf. Chr. Strosetzki, Grammatiker und Zensor im spanischen Siglo de Oro, dans Akten des Deutschen Hispanistentages Wolfenbüttel, 28.2.-1.3.1985; Schwerpunkt Siglo de Oro, Hans-Josef Niederehe (éd.), Hamburg 1986, p. 177-194. 
des livres recommandés et en se consacrant à l'enseignement de la morale, on peut découvrir chez $M^{\text {me }}$ de Maintenon une tentative didactique analogue. Les humanistes ont commencé en outre à séculariser la tradition médiévale des traités pour l'éducation d'un prince en l'adaptant, d'après l'éthique antique, aux différentes classes et vocations sociales. Ils élaborent ainsi des codes de conduite pour les juges, les médecins, les maires et aussi pour eux-mêmes. C'est l'Espagnol J. L. Vives qui, après avoir fait ses études à Paris, compose dans les Flandres en 1522/23 un traité sur l'éducation des filles ${ }^{5}$. Cet ouvrage, traduit en français vingt ans plus tard ${ }^{6}$, inspire Fénelon, qui peut transmettre à $M^{\text {me }}$ de Maintenon, grâce à son livre sur "L'éducation des filles », les idées de Vives.

Les codes de conduite et leurs critiques seront repris au XVII siècle par des écrivains moralistes, qui conçoivent l'honnête homme comme un courtisan mondain, ayant pour partenaire l'honnête femme. Les vertus morales de l'honnête femme ne sont pas encore remplacées par un art de plaire mondain et purement extérieur. Les œuvres de Jacques Du Bosc, "L'honnête femme " (1635), de Trotti de la Chétardie, «Instructions pour une jeune princesse, ou l'idée d'une honnête femme » (1684), et de l'abbé Gaussault, "le portrait d'une femme honneste, raisonnable et véritablement chrétienne » (1694), en témoignent ${ }^{7}$. Puisque c'est la nécessité de plaire qui prévaut à la cour et qui est traditionnellement réservée aux femmes, c'est à leur exemple que s'imposent les règles du comportement mondain et de la

5. De institutione feminae christianae (trad. esp. 1528); Fray Luis de León a pu se référer à Vives dans La perfecta casada (1583). Cf. Manfred Lentzen, "Vives' Ideen über die Erziehung der Frau. $\mathrm{Zu}$ "De institutione feminae christianae" " (1523), in Juan Luis Vives, Christoph Strosetzki (éd.), Frankfurt, Vervuert, 1995, p. 47-54 ; Joachin Leeker, "Das Frauenbild in Vives" De institutione feminae christianae und Castigliones Libro del cortegiano ", in ibid., p. 55-74.

6. "L'éducation de la femme chrétienne", traduit de l'espagnol par Pierre de Changy, J. Kerver, Paris 1543.

7. Chr. Strosetzki, Rhétorique de la conversation. Sa dimension littéraire et linguistique dans la société française du XVIr siècle, traduit en français par Sabine Seubert, Paris, Seattle, Tübingen 1984 (Biblio 17, 20), S. 139. 
conversation courtoise ${ }^{8}$. La question serait alors de savoir si $\mathrm{M}^{\text {me }} \mathrm{de}$ Maintenon, en accord avec les traités sur l'honnête homme, veut encourager les influences mondaines dans l'éducation ou bien si, avec le postulat de l'humaniste Vives, elle cherche à en restreindre la portée.

Les références sur la vertu chez $\mathbf{M}^{\text {me }}$ de Maintenon sont nombreuses. Elle dresse en effet dans les «Instructions aux Demoiselles de la classe bleue " (1705) un catalogue des quatre vertus justitia, prudentia, temperantia et fortitudo, toutes déjà connues à travers les traités pour l'éducation du prince sous le nom de vertus cardinales ${ }^{9}$, qu'elle fonde sur la religion et adapte à la situation de ses écolières. La justice devient alors un " désintéressement, qui nous rend capable de décider contre nous-mêmes en faveur de ceux qui ont le bon droit de leur côté"; la prudence, une "vertu qui règle toutes nos paroles et nos actions selon la raison et la religion "; et la tempérance est considérée comme une vertu essentielle pour l'instruction, "car le faible de la jeunesse est l'emportement pour la joie et le plaisir ; tout la met hors d'elle et l'empêche de se posséder, si elle n'a grand soin de retenir la fougue de ce penchant ${ }^{10}$. Ces efforts pédagogiques ne s'adressent pas à ceux qui cherchent à plaire conformément au postulat mondain, mais plutôt aux écolières «faisant plaisir dans ce qu'elles peuvent, ne fâchant personne, portant partout la paix, ne désunissant jamais, ne redisant que ce qui peut plaire et adoucir $\eta^{11}$. On ne cherche donc pas à plaire dans l'intention de montrer le meilleur de soi-même, mais en tenant au contraire le plus grand compte des vertus morales. Il est indispensable pour cela de présenter la vertu sous ses aspects les

8. «Ce n'est qu'en fréquentant les Dames, que nous acquérons cet air du monde, et cette politesse que nul conseil, ny aucune lecture ne peuvent donner. " Chalesme, $L$ 'homme de qualité, ou les moyens de vivre en homme de bien et en homme du monde, Amsterdam 1671, p. 197.

9. Cf. La conversation IX «Sur les quatre vertus cardinales": Justice, Force, Prudence, Tempérance, dans M. de Monmerqué (éd.), Conversations de Madame La Marquise de Maintenon, Paris 1828, $3^{\mathrm{e}}$ éd., J.-J. Blaise, p. 63 et suiv.

10. Lavallée, $M$. Th. (éd.), Lettres et entretiens sur l'éducation des filles par $M^{\text {me }}$ de Maintenon, $2^{\mathrm{e}}$ éd., t. 2, Charpentier, Paris 1861 , p. 105-110; dans la suite : « Lettres et entretiens ".

11. Lavallée, $M$. Th. (éd.), Lettres sur l'éducation des filles par $M^{\text {me }}$ de Maintenon, Charpentier, Paris 1854, p. 261 ; dans la suite : «Lettres». 
plus attrayants, en évoquant à cette occasion toutes les nuances sur l'honneur, la probité et la générositél ${ }^{2}$. L'objectif de l'éducation est de développer « des habitudes et un goût pour la vertu " ${ }^{13}$.

Nous pouvons d'ores èt déjà nous demander si $\mathbf{M}^{\text {me }}$ de Maintenon envisage à tous égards, tel que l'exigera plus tard le siècle des Lumières, l'égalité entre les pensionnaires de Saint-Cyr ${ }^{14}$, qu'elles soient maîtresses ou écolières, pauvres ou riches, nobles ou roturières ${ }^{15}$. Elle préconise un traitement plus ou moins sévère et indulgent selon le caractère et les capacités de chacune des élèves ${ }^{16}$. Une rigueur générale ne peut être profitable à toutes. Seule la patience sagement employée permet de rectifier les erreurs commises ${ }^{17}$. Bien que toutes reçoivent en principe la même attention pédagogique, une distinction est cependant faite selon le rang social des élèves : " Il faut donner à toutes même instruction sur la religion et sur les moeurs ; il faut un soin égal de leur santé ; mais je crois qu'il ne seroit pas mal à propos d'avoir une attention particulière à ouvrir et à former l'esprit de celles dont la qualité est plus illustre, quoique leur misère les réduise à Saint-Cyr ; leur naissance les rend capables de mariage, d'abbayes ou d'autres places considérables auprès des grands, [...] mais il faut que ces distinctions se fassent d'une manière imperceptible pour les autres $11^{18}$.

\section{Ibid., p. 25.}

13. Ibid., p. 244-245.

14. Prévot, J., La première institutrice de France, $M^{\text {me }}$ de Maintenon, Paris, 1981, Belin, p. 55 : " après avoir parlé (encore en 1715) de "la différence des conditions que Dieu a ainsi arrangées" (et qu'il convient donc de préserver), elle n'hésite pas en 1716 à recommander aux Dames de Saint-Cyr une éducation égalitaire, identique pour toutes. "

15. « Déjà dans le recrutement des pensionnaires de Noisy, on avait tendu à éliminer l'élément bourgeois. "Danielou, Madeleine, Madame de Maintenon éducatrice, Paris 1946, Bloud \& Gay, p. 75 ; cf. aussi « II est intéressant de remarquer que nous avons de $\mathrm{M}^{\text {me }}$ de Maintenon quatre programmes d'études différents. Le premier est adapté à des princesses, c'est celui qu'elle rédigea pour la duchesse de Bourgogne [...] Le second est dédié à des demoiselles nobles mais pauvres, celles de Saint-Cyr ; le troisième à des petites bourgeoises, [...]. Le quatrième programme d'éducation s'adresse à des enfants du peuple. [...] Ici, il ne s'agit plus que de travail manuel. " Ibid., p. 153-154.

16. Lavallée, M. Th. (éd.), Lettres et entretiens, $2^{e}$ éd., t. 1, p. 51.

17. Ibid., p. 388.

18. Lavallée, M. Th . (éd.), Lettres et entretiens, $2^{e}$ éd., t. 1, p. 38-39. 
Néanmoins, le principe d'équité reste valide. Puisque la noblesse a perdu en prestige et que dans le monde seuls l'habileté et l'argent comptent, pour $\mathrm{M}^{\mathrm{me}}$ de Maintenon ces injustices seront compensées par l'éducation. Les écolières de Saint-Cyr ont effectivement le privilège de bénéficier d'une bonne éducation, inaccessible aux jeunes filles de parents nobles et appauvris qui se voient souvent dans l'obligation de contraindre leurs propres enfants à s'engager comme domestiques chez autrui $^{19}$. De même que les écolières, les maîtresses de classes qui ont fait des vœux solennels doivent se plier délibérément au règlement ${ }^{20}$. Si l'on exige de chaque chrétien qu'il soit humble et modeste, ces deux qualités s'appliquent à plus forte raison aux femmes et particulièrement aux écolières de Saint-Cyr ${ }^{21}$.

\section{La cour, la piété et le devoir.}

Etant donné qu'il y a chez elle un respect des vertus, support de toute éducation, $\mathbf{M}^{\text {me }}$ de Maintenon affecte dans son jugement sur la vie de cour une grande réserve ${ }^{22}$. Elle fait elle-même l'expérience de l'«ennui qui dévore les grands, et la peine qu'ils ont a remplir leurs joumées $"^{23}$. Il est dit aussi dans la lettre LXI à $\mathrm{M}^{\text {me }}$ de Brinon, à propos de la duchesse du Maine, qu'elle est épuisée par les contraintes et par les fatigues de la cour ${ }^{24}$. Plutôt que de subir les intrigues de la cour, $\mathbf{M}^{\text {me }}$ de Maintenon veille à ce que les Dames de Saint-Cyr ne fassent pas prédominer l'esprit courtois voué aux apparences sur

19. Ibid., p. 340-341.

20. Ibid., p. 38-39.

21. Lavallée, M. Th. (éd.), Lettres, p. 90.

22. Danielou, M., Madame de Maintenon éducatrice, p. 44.

23. Gosse, Pierre - Luzac, Elie (éd.), Lettres de Madame de Maintenon, t. 2, La Haye, Leide 1941, p. 157. Cf. aussi Guevara de, Fr. Antonio, Menosprecio de corte y alabanza de aldea, M. Martínez de Burgos (éd.), Madrid 1915, p. 100 : « No hay en esta vida cosa que sea tan enemiga de la vertud, como es la ociosidad ; porque de los ociosos momentos y superfluos pensamientos tienen principio los hombres perdidos. "

24. "Sa coiffure pèse plus que toute sa personne. [...] Je voudrais la tenir à SaintCyr, vêtue comme l'une des vertes, et courant d'aussi bon cour. II n'y a point dans les couvents d'austérité pareille à celles auxquelles l'étiquette de la Cour assujettit les grands. "Ibid., p. 180. 
l'esprit initial de son institution ${ }^{25}$. Elle fait alors la distinction suivante : l'« esprit du côté du monde, du paganisme et des sentiments héroïques : c'est ce qu'il y a de plus opposé au christianisme, qui est humble et simple, et dans lequel on retrouve la véritable grandeur $12^{26}$. L'opposition, perçue entre le monde et la religion, rappelle alors l'antithèse de la courtoisie et de la vertu ${ }^{27}$. Une nouvelle question se pose donc : peut-on parler d'une éducation inspirée de la discipline ascétique des ordres religieux ? Saint-Cyr se distancie-t-il autant des couvents que de la cour ? Très tôt $\mathbf{M}^{\text {me }}$ de Maintenon est questionnée dans une lettre sur le caractère monastique de Saint-Cyr ${ }^{28}$.

$\mathrm{M}^{\mathrm{me}}$ de Maintenon fait de Saint-Cyr un établissement séculier, organisé selon les principes de la piété. Elle craint que le voisinage de la cour ${ }^{29}$, les visites du roi et l'éclat mondain d'une $\mathbf{M}^{\mathrm{me}}$ de Brinon $^{30}$ ne sécularisent exagérément l'esprit de la maison, en donnant aux écolières le sentiment de faire partie des grandes dames du monde ${ }^{31}$. Elle s'y oppose en instituant le vœu de pauvreté, qu'il fallait observer dans la fondation royale de la même manière que dans les couvents des

25. "A Saint-Cyr on a de la peine à revenir des premières idées, les maîtresses songeant plus à faire paroître leurs filles qu'à les rendre telles qu'on les voudroit. " Lavallée, M. Th. (éd.), Lettres, Paris, 1854, p. 222.

26. Lavallée, Th. (éd.), Lettres et entretiens, $2^{\mathrm{e}}$ éd., t. 1, p. 59.

27. "Qu'elles [les élèves] haïssent le monde, puisque notre Seigneur l'a haï, je dis même celles qui ont dessein d'y retoumer. "Lavallée, M. Th. (éd.), Lettres, S. 101 ; cf. aussi : P. Gosse, E. Luzac (éd.), Lettres, t. 3, p. 46-47.

28. "Faut-il des religieuses ou des séculières? La règle des religieuses peut-elle compatir avec les soins que demande l'éducation, sans avoir ni retraites ni offices particuliers ? Admettra-t-on la clôture entière ? Aura-t-on des soeurs converses ou des servantes ? Un seul prêtre suffit-il ? A quel âge rendrons-nous les demoiselles à leur parents? Si l'on ne veut pas de couvent, des voux simples suffisent-ils ? Combien de religieuses faudra-t-il pour Saint-Cyr [...]. Faites ce plan sans penser à votre intérêt, mais aussi sans oublier vos talents. " P. Gosse, E. Luzac (éd.), Lettres, t. 2, p. 135-136.

29. Danielou, M., Madame de Maintenon éducatrice, p. 95.

30. Ibid., p. 88. "Je voudrais que $\mathrm{M}^{\text {me }}$ de Brinon fût moins éloquente et plus régulière, qu'elle connût moins le monde et mieux les devoirs de son état, qu'elle fût moins visitée au dehors et plus accessible au dedans, qu'elle usât de plus de sévérité à l'égard d'elle-même et de plus d'indulgence à l'égard des autres. "

31. Lavallée, M. Th. (éd.), Lettres, p. 16. 
capucins $^{32}$. L'éducation ne prépare cependant pas à une vie monacale : "Qu'on les élève en séculières, bonnes chrétiennes, sans exiger d'elles des pratiques religieuses, comme de n'oser lever les yeux $n^{33}$. En effet, $\mathbf{M}^{\text {me }}$ de Maintenon se plaint à ce sujet, dans une lettre à $\mathbf{M}^{\text {me }}$ de Brinon, des Demoiselles qui, encore enfants, passent trop de temps à l'église ${ }^{34}$. Mais c'est certainement par complaisance pour $\mathbf{M}^{\mathrm{me}}$ de Brinon qu'il lui a fallu déplorer de tels abus ${ }^{35}$.

L'éducation prépare en principe les jeunes filles à occuper toutes les positions sociales possibles : "On leur inspire l'horreur du monde sans vouloir les contraindre à être religieuses ; mais on leur explique les avantages de cette condition. On les instruit des devoirs des femmes du monde et de tous les états où elles pourront se trouver $1{ }^{36}$. Membres de l'ordre de Saint-Louis, les maitresses qui ont prononcé leurs vœux et contracté ainsi un lien étroit avec l'école sont toutefois dispensées des exercices réguliers de la prière puisque leur tâche principale demeure l'éducation des Demoiselles ${ }^{37}$.

Selon $\mathbf{M}^{\text {me }}$ de Maintenon, deux choses opposées se côtoient dans l'esprit de ses écolières, qui parfois se tournent vers la vie intérieure et s'éloignent du monde, mais qui parfois posent aussi leur regard sur la vie extérieure et sur la réalité de ce monde. Et c'est cela qu'il faut éviter, car on aurait alors affaire à " une troupe de libertines qui s'ennuient, qui aspirent au monde, et qui ne soient arrangées qu'au dehors ${ }^{38}$. Bénéficiant à Saint-Cyr de liberté et d'un règlement particulier, les pensionnaires se doivent d'apprécier ce privilège : il faut que vous « leur fassiez sentir tout l'avantage du leur [de leur état] qui les sépare et les rend tout à fait indépendantes des intérêts du

32. Lavallée, Th. (éd.), Lettres et entretiens, $2^{\mathrm{e}}$ éd., t. 1, p. 144.

33. Lavallée, M. Th. (éd.), Lettres, p. 9-10.

34. Perkins, Wendy, "François de Sales, $M^{m e}$ de Maintenon and the uses of belief", in Elisabeth Fallaize (éd.), Representations of belief. Essays in memory of G. V. Banks, Rom Hallmark, Ian Pickup, Birmingham 1991, p. 56-57.

35. P. Gosse, E. Luzac (éd.), Lettres, t. 2, p. 141-142.

36. Lavallée, $M$. Th. (éd.), Lettres et entretiens, $2^{e}$ éd., t. 1, p. 10.

37. Lavallée, M. Th. (éd.), Lettres et entretiens, $2^{e}$ éd., t. 2, p. 385. Cf. aussi Danielou, M., Madame de Maintenon éducatrice, p. 166.

38. Lavallée, M. Th. (éd.), Lettres et entretiens, $2^{e}$ éd., t. 1, p. 125. 
monde ${ }^{39}$. La vie intérieure est donc définie comme un lieu de tranquillité et de travail éducatif. Il contraste en soi avec les éléments dérangeants de l'extérieur, rejetés dans l'intérêt de préserver une éducation constante et sereine ${ }^{40}$. Il faut donc laisser le monde extérieur hors des murs de Saint-Cyr. On donne bien sûr aux visiteurs mondains une impression générale d'ordre, et pour éviter qu'ils ne reviennent trop souvent, il est fori conseillé de ne pas éveiller leur intérêt. Mieux vaut les effrayer ${ }^{A 1}$. Toutes ces précautions doivent contribuer à préserver la paix et la sérénité à l'intérieur de l'école et à ne laisser pénétrer ni perturbation, ni danger ${ }^{42}$.

Quelles sont les règles de conduite observées par les Demoiselles de Saint-Cyr ? La vénération aussi bien du roi, fondateur de l'établissement, que de l'Eglise ${ }^{43}$, leur protectrice, constitue la première règle ${ }^{44}$. La morale chrétienne prend une place si importante qu'elle devient un modèle pour tout le pays : « Il y a donc dans l'œuvre de Saint-Louis, si elle est bien faite et avec l'esprit d'une vraie foi et d'un véritable amour de Dieu, de quoi renouveler dans tout le royaume la perfection du christianisme $"{ }^{45}$. Pour préparer les écolières aux devoirs de la vie civile, on leur impose une règle, établie selon la religion chrétienne. Il en va de même pour l'étiquette, car les écolières se contentent en effet d'apprendre «les bienséances que la religion approuve». Tout doit donc être envisagé «selon les lois de la

39. Ibid., p. 111-112.

40. Lavallée, M. Th. (éd.), Lettres, p. 251.

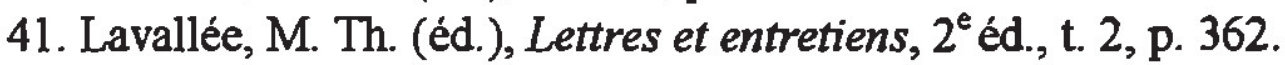

42. «Le secours au dedans doit se trouver dans les demoiselles [...] Avec tous ces secours, il faut n'en point faire venir du dehors, ce qui serait inégulier et dangereux. "Lavallée, M. Th. (éd.), Lettres, p. 203-206.

43. Guelfi, Julien, Madame de Maintenon 1635-1719, l'Hermès, Lyon 1986, p. 139. "Le 18 février 1690, le Pape Alexandre VIII écrivit à Madame de Maintenon une longue lettre. "

44. "Vous êtes indispensablement obligées de remplir l'intention du Roi votre fondateur, et de l'Eglise qui a supprimé un grand établissement pour fournir à l'éducation chrétienne d'un grand nombre de pauvres demoiselles. "Lavallée, $\mathrm{M}$. Th. (éd.), Lettres et entretiens, $2^{e}$ éd., t. 1, p. 137.

45. Ibid., p. 142. Cf. aussi Danielou, M., Madame de Maintenon éducatrice, p. 51. Cf. aussi Barbey d'Aurevilly, Jules, $A$ côté de la grande histoire, les auvres et les hommes XXI, Slatkine Reprints, Genève 1968, p. 29-30. 
philosophie du monde, mais rectifié par la piété et subordonné aux grandes règles du christianisme ${ }^{46}$. C'est la piété, accommodée de la docilité, qui forme les fondements du salut ${ }^{47}$. Tout en étant la base indispensable, la piété varie toutefois selon l'origine sociale et l'âge des Demoiselles. Car cela permet d'éviter une trop grande dévotion et les préserve de l'hypocrisie et du scrupule ${ }^{48}$. A cette grande piété s'accorde le vœu de pauvreté et de simplicité, de sorte que dans les ornements de l'église, des cloîtres, des jardins et dans le mobilier, c'est la simplicité qui honore Dieu et non la magnificence ${ }^{49}$.

\section{Servir, obéir et s'oublier soi-même}

La dépendance et la subordination sont deux principes dominants à Saint-Cyr. Issues de familles sans ressources, les écolières doivent être particulièrement redevables envers ceux qui leur ont permis d'accéder à l'éducation de Saint-Cyr. Il est écrit dans les "Usages des classes " (1686) qu'en réponse à une faveur reçue, les écolières garderont leur vie durant un sentiment de gratitude pour le roi et $\mathbf{M}^{\mathrm{me}} \mathrm{de}$ Maintenon ${ }^{50} . M^{\mathrm{me}}$ de Maintenon reconnaît trois qualités principales chez ses pensionnaires : "l'horreur du péché, la présence de Dieu et la docilité $\|^{51}$. L'enseignement de Saint-Cyr consiste à former essentiellement "des filles bien instruites, bien soumises et bien raisonnables $\prime^{52}$. Pour cela il faut que les maitresses de classe se montrent attentionnées envers leurs élèves, fassent appel à leur raison et les stimulent au moyen de l'émulation, de récompenses et de bonnes appréciations $^{53}$. Si les maîtresses ont à se plier au règlement et aux

46. Lavallée, M. Th: (éd.), Lettres, p. 146-147.

47. Ibid., p. 269. Cf. aussi Danielou, M., Madame de Maintenon éducatrice, p.133.

48. Lavallée, M. Th. (éd.), Lettres, p. 322.

49. P. Gosse, E. Luzac (éd.), Lettres, t. 3, p. 50. Cf. aussi Danielou, M., Madame de Matintenon éducatrice, p. 79.

50. Lavaliée, $M$. Th. (éd.), Lettres et entretiens, $2^{e}$ éd., t. 1, p. 16.

51. Lavallée, M. Th. (éd.), Lettres et entretiens, $2^{e}$ éd., t. 2, p. 38.

52. Lavallée, M. Th. (éd.), Lettres, p. 307.

53. Ibid., p. 307. Cf. aussi : " Ayez donc toujours pour fin le salut de vos filles; mais servez-vous tantôt de sévérité, tantôt de la douceur, et sans cesse de la patience." " Ibid., p. 170. 
décisions de leurs supérieurs, cela est aussi valable pour les écolières. $M^{\text {me }}$ de Maintenon justifie cette exigence en rappelant que de toute façon la femme est vouée par nature à l'obéissance. Toujours au service du prochain et dans un esprit de sujétion, la novice idéale doit constamment faire preuve d'humilité, d'obéissance pour parvenir par le renoncement à elle-même à la perfection chrétienne ${ }^{54}$. L'obéissance n'est pourtant pas pour $\mathbf{M}^{\text {me }}$ de Maintenon uniquement un devoir chrétien, elle est aussi la première vertu chez une femme. C'est en tant que telle qu'elle sera enseignée à Saint-Cyr, et fera partie plus tard des devoirs de l'écolière devenue adulte. Le plus grand art consiste à renoncer à son propre moi, à toujours se dominer et à se montrer docile envers celui dont on dépend. Puisque la femme est destinée à obéir et que cet engagement s'applique aussi bien aux maîtresses qu'aux écolières, il suffit d'après $\mathrm{M}^{\text {me }}$ de Maintenon d'accepter ce rôle avec joie. C'est le seul moyen pour la femme de parvenir à la paix et à la sécurité. Ainsi les maîtresses et leurs élèves deviennent l'exemple de l'obéissance et de la dépendance ${ }^{55}$.

L'obéissance signifie la subordination de sa propre personne à la volonté d'autrui ${ }^{56} . \mathrm{M}^{\text {me }}$ de Maintenon nie par conséquent le moi de la femme qui doit se fier à Dieu et non se reposer ni sur ses capacités, ni sur aucune perfection mondaine. Vouée à l'humilité, la femme devient ainsi le symbole de la grâce divine : "pour sanctifier les familles séculières et les couvents ; vous formerez d'excellentes vierges pour les cloîtres; et de pieuses mères pour le monde $1{ }^{57}$. L'altruisme difficile et douloureux, fondé sur la religion, ne peut s'acquérir que par l'éducation. $\mathbf{M}^{\text {me }}$ de Maintenon formule à travers une maxime la règle de conduite de la femme : «Votre sexe vous oblige à l'obéissance :

54. Lavallée, $M$. Th. (éd.), Lettres et entretiens, $2^{e}$ éd., t. 1 , p. 98 . Cf. aussi Perkins, W., François de Sales, p. 59-60.

55. "C'est encore là un endroit où je vous conjure d'être inébranlable; il est de l'ordre de Dieu d'obéir ; notre sexe est fait pour obéir ; votre état particulier est état d'obéissance, obéissez et obéissez avec joie, vous y trouverez la sûreté et la paix. " Lavallée, M. Th. (éd.), Lettres et entretiens, $2^{e}$ éd., t. 1, p. 120-125. On retrouve presque la même formule dans une autre lettre : Lavallée, M. Th. (éd.), Lettres, p. 57.

56. Cf. Danielou, M., Madame de Maintenon éducatrice, p.162.

57. Lavallée, $M$. Th. (éd.), Lettres et entretiens, $2^{\mathrm{e}}$ éd., t. 1, p. 141. 
vous ne sauriez trop vous y accoutumer. Ecoutez toujours et ne parlez guère. Dites toujours ce qui peut plaire, et jamais ce qui peut brouiller ${ }^{58}$. Les règles de l'obéissance et de la subordination valent aussi pour la vie conjugale. «Fuyez le monde. Attachez-vous à plaire à votre mari, et tâchez de ne plaire qu'à lui seul ${ }^{59}$. Cette sorte de complaisance obtient déjà aux yeux de $\mathbf{M}^{\text {me }}$ de Maintenon la valeur d'une prière. Elle résume ses conseils de la sorte : "Vous n'avez à présent, ma chère fille, que deux choses à faire : servir Dieu et plaire à votre mari $»^{60}$.

\section{Religion et travail, divertissement et utilité}

Comment $\mathbf{M}^{\text {me }}$ de Maintenon conçoit-elle la répartition du travail et de la prière dans la vie d'une femme ? Privilégiant au sens chrétien l'abnégation de sa personne, la femme selon $\mathbf{M}^{\text {me }}$ de Maintenon consacrera son temps et sa force au travail. "Soyez laborieuse : nous sommes tous nés pour le travail ; et aucun des moments de votre vie n'est à nous ${ }^{61}$. L'éducation, selon $\mathrm{M}^{\mathrm{me}}$ de Maintenon, s'accompagnera d'une nourriture simple, assurant une saine croissance, et préparera déjà à une vie de travail ${ }^{62}$. Suivant la règle bénédictine Ora et labora, qui n'est bien sûr pas toujours prise au pied de la lettre, le travail s'effectuera dans la prière ${ }^{63}$. "C'est une erreur ", dit-elle, " de croire que la vie intérieure ne consiste qu'à prier : elle consiste de plus à remplir les devoirs de son état et à travailler dans la vue de plaire à

58. Lavallée, M. Th. (éd.), Lettres et entretiens, $2^{\mathrm{e}}$ éd., t. 2, p. 411 ; cf. aussi « le portrait d'une personne propre à la société " : "elle veut tout ce qu'on veut jouer aux jeux que les autres proposent, quand ils ne seraient pas de son goût ", p. 5 ; la personne qui trouble la société : "elle oublie les autres", M. de Monmerqué (éd.), Conversations, p. 6.

59. P. Gosse, E. Luzac (éd.), Lettres, t. 2, p. 191-192.

60. Ibid., p. 194-196.

61. Ibid., p. 191-192.

62. "Nourrissez-les durement, accoutumez-les à toutes sortes de fatigues : elles sont pauvres, et apparemment elles le seront toujours; élevez-les donc dans l'état où il a plu à Dieu de les mettre ; mais n'oubliez rien pour sauver leur âme, pour fortifier leur santé, et pour conserver leur taille. " Lavallée, M. Th. (éd.), Lettres, p. 198.

63. Cf. Prévot, J., La première institutrice de France, p. 46. 
Dieu $)^{64}$. Etant donné que $\mathrm{M}^{\mathrm{me}}$ de Maintenon retient principalement de la vie chrétienne son côté pratique, les bonnes actions priment sur les visites à l'église. A ce propos, elle raconte qu'on se moquait d'une fille de couvent qui prétendait qu'il ne fallait en aucun cas "perdre vêpres " et félicite par contre celle qui, pour tenir compagnie à un mari malade, s'abstient d'y aller. Qui préfere, plutôt que d'aller à la messe tous les matins, élever ses enfants et donner des instructions à ses domestiques, mérite le respect et l'honneur ${ }^{65}$. Il faut donc privilégier " une religion solide et [sa mise] en pratique $\aleph^{66}$. L'intérêt porté à la vie pratique ${ }^{67}$ et l'importance accordée à l'utilité sont bien la preuve qu'il y avait déjà à Saint-Cyr les signes avant-coureurs d'une pensée qui ne verra son épanouissement qu'au siècle des Lumières. $\mathbf{M}^{\text {me }}$ de Maintenon souhaite faire de sa maison un modèle qui puisse inciter les puissants à "multiplier ces établissements utiles ${ }^{68}$. Ce qui est caractéristique ici, c'est que l'on insiste sur l'utilité de l'école en tant qu'institution. Par conséquent, il faut y enseigner des matières utiles comme par exemple l'écriture et la lecture de textes français, le calcul, la religion et le métier de servir ${ }^{69}$. Pour les religieuses, les cours d'histoire ne reposent que sur "une légère connoissance" des noms de princes et de souverains français et étrangers, pour " n'être pas plus ignorante que le commun des honnêtes gens ${ }^{70}$.

Le divertissement chez $\mathrm{M}^{\mathrm{me}}$ de Maintenon n'est plus au centre de l'intérêt comme pour l'honnête femme mondaine ${ }^{71}$. Il est tout juste un moyen pédagogique. Le motif présenté, pour encourager l'activité théâtrale à Saint-Cyr, le prouve bien. L'éducatrice veut divertir ses filles avec de belles choses, en leur enseignant à bien prononcer, les retirant ainsi "dé la conversation qu'elles ont entre elles $»^{7}$. Un

64. Lavallée, M. Th. (éd.), Lettres et entretiens, $2^{e}$ éd., t. 2, p. 211.

65. Lavallée, M. Th. (éd.), Lettres, p. 311.

66. Lavallée, M. Th. (éd.), Lettres et entretiens, $2^{\mathrm{e}}$ éd., t. 2, p. 291.

67. Cf. Danielou, M., Madame de Maintenon éducatrice, p.173.

68. P. Gosse, E. Luzac (éd.), Lettres, t. 2, p. 135.

69. Lavallée, M. Th. (éd.), Lettres, p. 2.

70. Lavallée, M. Th. (éd.), Lettres et entretiens, $2^{e}$ éd., t. 1, p. 223.

71. Cf. Danielou, M., Madame de Maintenon éducatrice, p. 116-117.

72. Lavallée, M. Th. (éd.), Lettres, p. 215. 
divertissement employé à des fins pédagogiques est donc judicieux puisqu'il est censé interrompre le bavardage inconsistant des élèves.

\section{Conversation, politesse mondaine et silence}

La vie mondaine, qui au siècle de Louis XIV joue un rôle décisif à la cour et se caractérise par la représentation et la bienséance, place la femme au centre de toute conversation, ce que refuse $\mathbf{M}^{\text {me }}$ de Maintenon. «Ne croyez donc point, mes chères soeurs, avoir à soutenir les bienséances d'une fondation royale ; rejetez le nom de Dames ; prenez plaisir à vous appeler les filles de Saint-Louis ; ayez un air simple, religieux et monastique, contraire à toutes les politesses mondaines $n^{7}$. Elle souligne plus loin son refus de la bienséance courtoise en définissant la civilité, non pas selon les règles de l'apparence, mais selon un principe éthique, qui désavoue l'amourpropre dans les relations sociales. Et elle écrit à ce propos : "Pour ce qui regarde la société, je ferois consister la civilité à s'oublier soi-même pour s'occuper de tout ce qui convient aux autres ${ }^{74}$. La civilité désigne donc dorénavant le respect d'autrui, l'abnégation de sa propre personne, le refus de tout ce qui peut incommoder son prochain, tel le fait par exemple de ne parler que de soi, de ne pas écouter son interlocuteur assez longtemps ou de ne pas s'éloigner d'un groupe de personnes qui parlent à voix basse.

Les règles de bienséance, envisagées de la sorte, n'appartiennent plus au domaine de l'étiquette, mais à celui de l'estime et du ménagement d'autrui, qui confêrent à ces mêmes règles cette fois-ci une valeur éthique. Pour soulager les cas de conscience et consolider les principes religieux, $\mathbf{M}^{\text {me }}$ de Maintenon prône la simplicité, la bonne foi et la droiture, en condamnant par contre le raffinement mondain et l'ostentation. "Montrez la haine que vous avez pour les finesses, pour les jugements, enfin pour tous les mauvais caractères d'esprit, qu'il faut tâcher de détruire dès l'enfance ${ }^{75}$. En même temps que la finesse,

73. Lavallée, Th. (éd.), Lettres et entretiens, $2^{e}$ éd., t. 1, p. 155.

74. Ibid., p. 368 ; cf. aussi « la grande politesse est de ménager en tout et partout les gens avec qui nous vivons. " M. de Monmerqué (éd.), Conversations, p. 3.

75. Lavallée, M. Th. (éd.), Lettres, p. 196. 
elle rejette l'habileté et la souplesse dans l'art de la conversation : " il faut abandonner l'éloquence, qui pourroit attirer l'attention des auditeurs $[\ldots] \|^{76}$. Elle refuse aussi dans ce contexte le caractère spirituel du bel esprit qui, s'il est ridicule pour le monde profane, est vil et démoniaque dans le monde chrétien. Il suffit de rappeler l'éloge que $\mathbf{M}^{\mathrm{me}}$ de Maintenon fait d'une novice, au comportement exemplaire : " elle ne songe point à montrer de l'esprit ; elle tourne la conversation selon le goût des autres; si on l'interrompt quand elle a commencé un discours, elle ne pense pas même à le reprendre, à moins qu'on ne lui demande, parce que dans les plus petites choses, comme dans les plus grandes, elle ne veut tenir à rien ${ }^{77}$.

Flattant l'amour-propre et consolidant la position sociale de chacun, les relations mondaines favorisent l'égocentrisme et font donc l'objet de nombreuses critiques : "L'effectif est de ne point voir les gens du monde, c'est de n'être point vues, c'est d'être ignorées, oubliées, anéanties dans une mort civile ; c'est de ne plus entendre. [...] Le silence est le plus grand remède à l'infirmité humaine dans une communauté : on peut dire que c'est le supplément de la plus parfaite vertu. [...] il faut renoncer au bel esprit, il est ridicule dans le monde profane, affreux et diabolique dans les vierges de Jésus-Christ ${ }^{78}$. La présence des femmes dans la société n'est plus conditionnée par l'art de la conversation, mais par l'obligation de se taire. Le silence devient alors synonyme de subordination ; il est comme ce garde-fou qui servirait d'obstacle à la prétention et préserverait contre les actes inconsidérés. Le conseil donné à $\mathbf{M}^{\text {me }}$ de Glapion, au sujet de l'éducation des Demoiselles, illustre bien cela : «ne les rendez pas trop discoureuses, ne leur inspirez pas le goût de la conversation $"$ ". Il faut donc les exhorter au silence et à la religion. La discrétion est de rigueur dans toutes les conversations et particulièrement dans celles qui traitent de sujets importants : «ne rendez point nos filles des discoureuses

76. Ibid., p. 22.

77. Lavallée, M. Th. (éd.), Lettres et entretiens, $2^{\mathrm{e}}$ éd., t. 1 , p. 102 ; cf. aussi la "différence qu'il y a entre avoir de l'esprit et un bon esprit ", M. de Monmerqué (éd.), Conversations, p. 31

78. Lavallée, $M$. Th. (éd.), Lettres et entretiens, $2^{\mathrm{e}}$ éd., t. 1, p. 152-154.

79. Lavallée, M. Th. (éd.), Lettres, p. 228. 
spirituelles : rien n'est plus dangereux et plus inutile; qu'elles ne lisent guère ; qu'elles parlent peu sur les matières relevées : qu'elles gardent leurs règles ; qu'elles s'éloignent du monde ; qu'elles travaillent de leurs mains ; voilà le moyen d'avoir une communauté régulière $\|^{80} . \mathrm{M}^{\mathrm{me}}$ de Maintenon qualifie de peu vertueux le talent des discoureuses que vantaient les traités sur la conversation au $\mathrm{XVII}^{\mathrm{e}}$ siècle. Elle préfêre la règle du silence, très utile aux femmes, qui par nature sont " portées à beaucoup parler $~{ }^{81}$, et qu'il faut habituer très tôt au silence. Il faut aussi éviter la conversation parce qu'elle est synonyme d'oisiveté. Les Demoiselles de la maison de Saint-Louis seront «silencieuses et laborieuses ${ }^{82}$, afin de remplir leurs devoirs de femmes.

\section{La curiosité et la lecture}

Si la conversation enjouée, considérée comme un divertissement, est interdite, la conversation sérieuse l'est encore plus, et cela pour deux raisons : c'est en la pratiquant que les Demoiselles affirment leur bon esprit, mais c'est elle aussi qui incite à cette curiosité dangereuse qui serait susceptible de les éloigner du droit chemin. Il faut donc interdire « la liberté de faire des questions inutiles et curieuses qui ne servent point à former leur raison et leur moeurs ${ }^{83}$. On évitera que les filles deviennent des frivoles discoureuses qui posent des questions multiples pour le seul plaisir de parler. Il faudra leur défendre de ne songer qu'à un divertissement qui les détourne du vrai but que se propose leur éducatrice. Si l'éducation des jeunes filles consiste avant tout à foumir de solides bases pour leur future vie sociale, le reste ne compte guère. "Ne donnez rien à leur curiosité, empêchez les grands raisonnements, raffinements, objections, et tâchez de démêler si c'est la vérité qu'elles cherchent ou si elles veulent disputer pour se divertir, pour embarrasser ou pour montrer leur esprit $n^{84}$. Quand elle n'est pas dictée par le désir de simplicité, la curiosité est blâmable. Elle conduit

80. P. Gosse, E. Luzac (éd.), Lettres, t. 3, p. 35-36.

81. Lavallée, M. Th. (éd.), Lettres, p. 93.

82. Lavallée, $M$. Th. (éd.), Lettres et entretiens, $2^{e}$ éd., t. 1, p. 10.

83. Lavallée, M. Th. (éd.), Lettres et entretiens, $2^{e}$ éd., t. 2, p. 228.

84. Lavallée, M. Th. (éd.), Lettres, p. 39. 
en effet au raffinement et à l'étalage de l'esprit et alimente les premiers doutes sur l'orthodoxie. $\mathbf{M}^{\text {me }}$ de Maintenon se méfie donc de cet élargissement de connaissances qui va de pair avec la lecture. Elle cherche à limiter les livres et à les contrôler en instituant un canon d'ouvrages sélectionnés et recommandés et en refusant les autres, considérés comme pernicieux et excitant « une curiosité insatiable ${ }^{85}$.

Les Dames sont à la fois maîtresses et censeurs chargés de diriger les lectures de leurs élèves. Elles leur donnent des directives herméneutiques sur l'exégèse claire et simplifiée de la Bible, en privilégiant la mise en pratique des lectures. «Qu'on se rendît maître de leurs lectures et qu'on leur dit que dans le Nouveau Testament il faut adorer ce qu'on n'entend pas et pratiquer ce qu'on entend $1{ }^{86}$. Pour ne pas s'exposer au danger des mauvais livres, $M^{\text {me }}$ de Maintenon conseille de choisir de bons livres qu'on relira plusieurs fois : " Ayez peu de livres, et des plus communs, et surtout n'en lisez jamais de suspects, ni pour la doctrine ni pour les moeurs ; lisez ceux que vous avez bien choisis, cinquante fois, cent fois, si vous voulez, et enfin jusqu'à ce que vous ayez mis en pratique ce qu'ils enseignent, car c'est la fin que nous devons nous proposer dans toutes nos lectures spirituelles ${ }^{87}$. Une fois de plus la lecture est envisagée dans ses applications directes. Peu importe la qualité ou la profondeur des connaissances, il suffit de pouvoir les transposer dans la vie concrète d'une femme. Cela explique l'importance accordée à l'étude circonstanciée des maximes traitant de la bonne conduite, ainsi que la méfiance entretenue vis-à-vis de la diversité des livres. $\mathbf{M}^{\text {me }}$ de Maintenon composait elle-même de nombreuses sentences, qu'elle conseillait d'utiliser ${ }^{88}$. Elle recommandait aussi aux Dames de SaintLouis de méditer sur les maximes de saint François de Sales, comme par exemple : "de parler peu et de faire beaucoup $»^{89}$. Chargées de

85. P. Gosse, E. Luzac (éd.), Lettres, t. 3, p. 17.

86. Lavallée, M. Th. (éd.), Lettres et entretiens, $2^{\mathrm{e}}$ éd., t. 1, p. 3.

87. Ibid., p. 193.

88. "Ne point passer de jour sans avoir donné une bonne maxime à votre classe." " Lavallée, M. Th. (éd.), Lettres et entretiens, $2^{e}$ éd., t. 2, p. 64. Cf. aussi Danielou, M., Madame de Maintenon éducatrice, p. 181.

89. Lavallée, M. Th. (éd.), Lettres, p. 148. 
conseils pratiques sur le comportement à observer dans la vie quotidienne, les maximes ont une valeur pédagogique importante. Une différence est d'ailleurs faite entre les maximes religieuses et celles « de l'honneur et de la bienséance ${ }^{90}$.

\section{Censure et instruction canonique}

Le choix des livres doit être restreint. En plus de la Bible, $\mathrm{M}^{\mathrm{me}} \mathrm{de}$ Maintenon recommande la lecture attentive de livres exclusivement religieux : "Ne souffrez pas une grande diversité de livres. Le Nouveau Testament, l'Imitation, Grenade, Rodriguez, saint François de Sales et quelques autres, suffisent pour toute la vie d'une personne $n^{91} . \mathbf{M}^{\mathrm{me}} \mathrm{de}$ Maintenon rappelle fréquemment le rôle important de la censure à Saint-Cyr. Elle tolère avec vigilance la présence de livres dans l'établissement, mais prend garde cependant "de n'en laisser entrer aucun qui n'ait été examiné et approuvé par vos supérieurs $»^{92}$. Cela vaut aussi bien pour les livres que pour les manuscrits. Nuisibles sont les livres qui enfreignent les bonnes moeurs et répandent l'hérésie religieuse. On ferme pour cette raison à Saint-Cyr la bibliothèque aux œuvres quiétistes de $\mathbf{M}^{\text {me }}$ Guyon, Les torrents, Moyen court et $L$ 'explication du cantique des cantiques ${ }^{93}$.

De même que certains livres, les préceptes inutiles sont aussi défendus. $M^{\text {me }}$ de Maintenon qualifie de dangereux tous les livres profanes. Ils sont particulièrement nocifs pour les femmes puisqu'ils renforcent chez elles une curiosité qui leur est innée. Elle différencie, selon le genre des livres, plusieurs risques. Pernicieux sont à ses yeux les romans, dans lesquels il n'est question que de passions et de vices. Moins dangereux sont les livres sur l'histoire de la Rome antique ou sur l'histoire universelle, bien qu'ils détoument de la lecture d'ouvrages de piété et éveillent l'outrecuidance. Elle écrit à ce sujet : "J'appelle livres profanes, [...] tous ceux qui ne sont pas pieux, quoiqu'ils soient

90. Lavallée, M. Th. (éd.), Lettres et entretiens, $2^{\mathrm{e}}$ éd., t. 2, p. 174.

91. Lavallée, M. Th. (éd.), Lettres, p. 41.

92. Lavallée, M. Th. (éd.), Lettres et entretiens, $2^{\mathrm{e}}$ éd., t. 2, p. 239.

93. " J'ai bien prié madame notre Supérieure de ne plus mettre ces livres entre les mains de nos Dames. Cette lecture est trop forte pour elles : il leur faut un lait proportionné à leur âge. " P. Gosse, E. Luzac (éd.), Lettres, t. 2, p. 105. 
innocents, dès qu'ils n'ont pas une vraie utilité ${ }^{94}$. Pour ne pas prendre goût à la lecture, les filles doivent être élevées dans un esprit de travail. On substitue donc à la lecture les travaux domestiques, les ouvrages d'aiguilles et autres tâches propres aux devoirs d'une femme ${ }^{95}$. Ce qui compte pour une femme, ce n'est pas d'être savante ni héroïque, mais de s'occuper avec soin de sa maison. En outre, pour $\mathrm{M}^{\mathrm{me}}$ de Maintenon, ce que savent les femmes, elles ne le savent qu'à moitié, et le peu qu'elles savent les rend prétentieuses, bavardes et fières. L'approfondissement des connaissances ne convient pas au rôle de la femme ${ }^{96}$. Puisque la curiosité en général ne correspond pas à une vertu scientifique, mais plutôt à un vice féminin, il faut l'extirper du cœur des jeunes filles.

"Il suffit qu'elles sachent écrire pour faire leurs mémoires et leurs comptes ; il ne faut pas leur apprendre à faire de lettres ni parler de style : un peu d'orthographe leur suffit ; il n'en est pas de même de l'arithmétique, elle leur est nécessaire $1{ }^{97}$. Le choix surveillé des livres et la restriction du savoir servent finalement à conserver les rapports établis, empêchant les femmes d'évoluer dans leur position sociale. "Prêchez-leur la modération ; qu'il ne faut pas que le paysan fasse le bourgeois, ni que le bourgeois fasse le gentilhomme $1^{98}$. Dans un autre extrait, $\mathbf{M}^{\text {me }}$ de Maintenon communique sa définition de l'éducation bourgeoise : " Il faut élever vos bourgeoises en bourgeoises ; il ne leur faut ni vers, ni conversations ; il n'est point question de leur orner l'esprit $\|^{99}$. Bien plutôt, elles doivent s'appliquer à exercer vertueusement leurs obligations en tant qu'épouse et mère, intendante de leur foyer et, le dimanche et les jours de fête, fidèle de la paroisse.

\section{Nouveauté, christianisme et raison}

L'attitude subjective de la curiosité correspond dans le contexte historique à des idées religieuses nouvelles qui apparaissent portées par

94. Lavallée, M. Th. (éd.), Lettres et entretiens, $2^{e}$ éd., t. 1, p. 225-227.

95. Prévot, J., La première éducatrice de France, p. 35.

96. Perkins, W., François de Sales, p. 55.

97. Lavallée, M. Th. (éd.), Lettres, p. 322. Lettre à $\mathbf{M}^{\text {me }}$ de La Mairie (1713).

98. Ibid., p. 322.

99. Ibid., p. 314. 
les courants hérétiques modernes du jansénisme et du quiétisme. Selon $\mathrm{M}^{\mathrm{me}}$ de Maintenon, c'est un avantage qu'ont les femmes de ne pas avoir à se prononcer dans le débat religieux. L'ignorance garantit ainsi le droit chemin et l'obéissance à l'égard de l'Eglise catholique ${ }^{100}$. La recherche de la nouveauté est l'argument dont se servent les hérétiques pour convaincre ${ }^{101}$.

Les femmes doivent se méfier des vices, dans lesquelles on reconnaît d'après $\mathbf{M}^{\mathrm{me}}$ de Maintenon l'hérésie des faux prophètes. "Vous la reconnoîtrez à la nouvauté, à la singularité, à la désobéissance, à l'entêtement, au soin de se cacher aux supérieurs, aux troubles et aux autres excès qu'elle produira ${ }^{102}$. Celui qui adopte les vraies idées de la simplicité et de l'humilité et refuse toute sorte de présomption, de vaine politesse et curiosité, est à l'abri de l'hérésie. En obéissant aux règles religieuses et en se soumettant à l'autorité supérieure, la femme gagne aux yeux de $\mathrm{M}^{\text {me }}$ de Maintenon une sûreté bienfaisante et le bonheur de ceux qui se plient volontiers aux principes imposés. "C'est aux docteurs à raisonner et à approfondir ; celui quiles fait docteurs leur donne les lumières dont ils ont besoin $»^{103} . \mathrm{M}^{\mathrm{me}} \mathrm{de}$ Maintenon qualifie de prétentieux les calvinistes et les jansénistes qui prétendent, en s'opposant au dogme catholique, qu'il suffit de lire la Bible et de s'en tenir à son propre jugement. Pour elle au contraire, " la

100. Lavallée, $M$. Th. (éd.), Lettres et entretiens, $2^{\mathrm{e}}$ éd., t. 2, p. 363-364. Cf. aussi Perkins, W., François de Sales, p. 62.

101. "Si les jansénistes et les quiétistes vous connoissoient ce foible, ils auroient bientôt trouvé le secret de vous débiter toutes leurs erreurs par de petits imprimés, des maximes, des sentences qui se vendent presque pour rien, des cantiques nouveaux et spirituels. C'est dans ces sortes de livres qu'on trouve les choses les plus dangereuses, parce qu'ils ne sont pas sujets à l'examen et à la censure. * Lavallée, M. Th. (éd.), Lettres et entretiens, $2^{\mathrm{e}}$ éd., t. 2, p. 240.

102. Lavallée, $M$. Th. (éd.), Lettres et entretiens, $2^{\mathrm{e}}$ éd., t. 1, p. 157-158.

103. Lavallée, $M$. Th. (éd.), Lettres, p. 328. A un degré plus élevé le roi fait aussi partie des supérieurs : «Le Roi est plein de bons sentiments : il lit quelques fois l'Ecriture sainte : et il trouve que c'est le plus beau de tous les livres. I avoue ses faiblesses : il reconnoit ses fautes; il faut attendre que la grâce agisse. II pense sérieusement à la conversion des hérétiques; et dans peu on y travaillera tout de bon. " P. Gosse, E. Luzac (éd.), Lettres, t. 2, p. 82. 
vérité est simple, humble, elle veut de la dépendance pour marcher avec sûreté $\eta^{104}$.

Lorsque $\mathrm{M}^{\text {me }}$ de Maintenon se prononce contre les raffinements de la politesse mondaine, le bel esprit et la connaissance acquise au fil de nombreuses lectures, elle défend malgré tout une qualité intellectuelle, selon laquelle elle oriente son éducation. Lorsqu'elle propose : «On doit moins songer à orner leur esprit qu'à former leur raison $\eta^{105}$, elle s'oppose aux connaissances inutiles et donne de l'importance à la force du jugement. Il ne faut pas surcharger la mémoire de connaissances, mieux vaut former la raison, plus utile pour s'orienter dans la vie pratique ${ }^{106}$.

Il serait cependant erroné de voir dans l'importance très souvent accordée à la raison déjà le recours au message exprimé également au siècle des Lumières ${ }^{107}$. Pour $\mathrm{M}^{\text {me }}$ de Maintenon, être raisonnable, c'est avant tout être respectueux et obéissant. C'est dans ce contexte qu'il faut comprendre le propos : seule est raisonnable celle qui partage cet avis. Nous sommes bien loin de la raison du siècle des Lumières. Ici, raisonnable est donc pris dans le sens d'obéissant ${ }^{108}$. Il importe avant tout de "former la piété, la raison et les moeurs de vos filles" ${ }^{109}$. La plupart des Demoiselles de Saint-Cyr doivent être éduquées sans grande prétention, "mais le christianisme et la raison, qui est tout ce que l'on veut leur inspirer, sont également bons aux princesses et aux misérables $\eta^{110}$. Le mot raison s'emploie donc dans de similaires associations, telles que : «Il me semble que les Demoiselles entendent mieux raison que jamais, qu'elles deviennent plus humbles, plus

104. Lavallée, M. Th. (éd.), Lettres et entretiens, $2^{e}$ éd., t. 2, p. 315.

105. Lavallée, M. Th. (éd.), Lettres, p. 23. Lettre aux dames de Saint-Louis, sur l'éducation des Demoiselles, (1686).

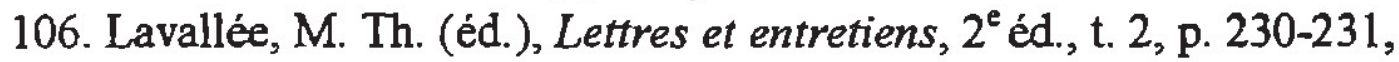
107. «Les vertus ont besoin de la raison pour agir à propos, et pour ne prendre nulle extrémité ", p. 12 ; la raison " vient de Dieu, qui veut bien être appelé la souveraine Raison ", M. de Monmerqué (éd.), Conversations, p. 13

108. "Vous qui êtes si bien instruites, à qui on tâche d'apprendre sitôt à obéir, obéissez donc, soumettez-vous, rien n'est meilleur, c'est le partage de notre sexe [...] Vous ne trouverez personne sur la terre de raisonnable qui ne se soumette." Lavallée, M. Th. (éd.), Lettres et entretiens, $2^{e}$ éd., t. 2, p. 251.

109. Lavallée, M. Th. (éd.), Lettres et entretiens, $2^{e}$ éd., t. 2, p. 172.

110. Lavallée, M. Th. (éd.), Lettres, p. 26. 
honnêtes, plus soumises, plus reconnoissantes envers la communauté " $"$ "111 ou bien encore : "Soyez chrétienne et raisonnable, ayez une piété sincère, libre, gaie, ronde, sans raffinement, comme de bonnes séculières; vous n'en serez par la suite que meilleures religieuses, si Dieu vous y appelle $\aleph^{112}$. Etre raisonnable n'aboutit donc pour $\mathbf{M}^{\mathrm{me}}$ de Maintenon ni à la mise en doute des anciens préjugés grâce à l'acquisition de connaissances nouvelles, ni à la remise en question des vieilles traditions. Bien au contraire, être raisonnable, c'est se plier à une orthodoxie traditionnelle pour éviter les dangers d'idées nouvelles menaçant de rompre avec la tradition ${ }^{113}$.

En résumé, les idées de $\mathbf{M}^{\text {me }}$ de Maintenon sont par conséquent plus proches de l'humanisme de Vives que de la curiosité de Montaigne. Elle oppose, dans un code de recommandations, les lectures utiles et celles qui ne satisfont que la soif d'apprendre. Elle préfere donc à une multitude de livres inutiles un tout petit nombre d'ouvrages utiles. A son avis il est préférable de rester dans l'ignorance car les connaissances nouvelles sont sources d'erreurs. Enfin, pour $\mathbf{M}^{\text {me }}$ de Maintenon, la raison n'est pas le jugement critique porté sur les préjugés transmis par la tradition, comme elle le sera plus tard au siècle des Lumières. Elle est ce qui permet d'admettre que l'obéissance est une chose nécessaire.

En appuyant son œuvre éducative sur des écrits et des directives, elle partage les exigences des humanistes. Elle ébauche le modèle complexe d'une élève, donc d'une femme, qui est diamétralement opposé au concept de l'honnêteté développé dans les traités moraux sur la conversation au $X V \mathrm{II}^{\mathrm{e}}$ siècle. C'est grâce à la piété et à une morale fondées sur les vertus cardinales que le principe de plaire s'élève au niveau éthique du respect envers autrui. Saint-Cyr se distingue à la fois de la vie dans les couvents et de la vie à la cour, qui est considérée comme épuisante et incompatible avec une vie religieuse. Saint-Cyr apparaît comme un lieu d'éducation, scrupuleusement protégé des attaques et influences extérieures. La piété, l'obéissance et la

111. Ibid., p. 212.

112. Ibid., p. 295 ; cf. aussi Lavallée, M. Th. (éd.), Lettres et entretiens, $2^{\mathrm{e}}$ éd., t. 2, p. 145.

113. Danielou, M., Madame de Maintenon éducatrice, p. 155. 
soumission font partie des devoirs de la vie civile. Le moi propre sera donc dédaigné, voire rejeté. La soumission féminine, exprimant dans son origine religieuse l'humilité face à Dieu et dans son origine sociale la dépendance de l'épouse vis-à-vis de son mari, est approuvée à SaintCyr comme un principe indispensable. Lorsque $\mathbf{M}^{\text {me }}$ de Maintenon choisit la piété et loue pour cela l'esprit de pratique, le travail et l'utilité, elle rejette aussi en même temps la théorie, la trop grande dévotion et le divertissement. Lorsqu'elle s'oppose aux principes de la société courtoise, elle se réfêre aux postulats de la Renaissance et du siècle des Lumières. En se dressant contre le bel esprit, les finesses mondaines, l'éloquence des gens du monde et le goût pour la conversation des filles discoureuses et curieuses, et en revendiquant à leur place la règle du silence, $\mathbf{M}^{\text {me }}$ de Maintenon confirme son désaccord avec le style de vie courtoise. Tout en rappelant les valeurs chrétiennes et en intériorisant un comportement extérieur nouvellement défini, elle s'approprie d'une manière tout à fait personnelle le jugement porté par le siècle des Lumières sur les traditions de la société actuelle. C'est pourquoi sa critique de la cour ne rappelle pas uniquement les préoccupations des humanistes, mais semble aussi anticiper sur l'œuvre du XVIII ${ }^{e}$ siècle. Educatrice, $\mathbf{M}^{\text {me }}$ de Maintenon se soucie avec le même intérêt que les humanistes des questions centrales de la forme et des valeurs du comportement humain, ainsi que de la place réservée à la femme dans la société. 